\title{
Fatty acid synthase expression in osteosarcoma and its correlation with pulmonary metastasis
}

\author{
ZHI LI LIU ${ }^{1 *}$, GAO WANG ${ }^{2 *}$, AI FEN PENG ${ }^{3}$, QING FENG LUO ${ }^{4}$, YANG ZHOU ${ }^{1}$ and SHAN HU HUANG ${ }^{1}$ \\ ${ }^{1}$ Department of Orthopedics, First Affiliated Hospital of Nanchang University; ${ }^{2}$ Department of Orthopedics, \\ Third Affiliated Hospital of Nanchang University; ${ }^{3}$ Jiangxi University of Traditional Chinese Medicine, Nanchang; \\ ${ }^{4}$ Department of Orthopedics, The Second People's Hospital of Jiangxi Province, Jiangxi, P.R. China
}

Received April 16, 2012; Accepted July 27, 2012

DOI: $10.3892 / \mathrm{ol} .2012 .862$

\begin{abstract}
Previous experimental evidence has suggested that fatty acid synthase (FASN) may be involved in cancer metastasis. However, its role has been poorly evaluated in osteosarcoma. The aim of this study was to investigate the correlation of FASN expression with pulmonary metastasis and the correlation of FASN expression with the Ki-67 antigen, a proliferation marker, in patients with osteosarcoma of the extremities. The expression of FASN protein and Ki-67 was detected by immunohistochemistry of biopsy tissues from 136 patients with osteosarcoma of the extremities and 21 cases of osteoenchondroma. Positive expression of the FASN protein was observed and located in the cytoplasm. The positive expression rate of FASN was $63.2 \%$ in osteosarcoma and $28.6 \%$ in osteoenchondroma $(\mathrm{p}<0.05)$. The expression levels of the FASN protein were higher in the cases with lung metastasis compared to those without metastasis $(\mathrm{p}<0.01)$. The percentage of Ki-67 stained nuclei in osteosarcoma with pulmonary metastasis and in those without was $43.43 \pm 10.05$ and $25.41 \pm 6.68 \%$, respectively $(\mathrm{p}<0.01)$. There was a positive correlation between FASN and $\mathrm{Ki}-67$ protein expression in osteosarcoma (Spearman's rho, $\mathrm{F}=43.05, \mathrm{R}=0.734$ ). Therefore, FASN may be a promising target in the treatment of osteosarcoma metastasis.
\end{abstract}

\section{Introduction}

Osteosarcoma (OS) is the most common tumor in bone and the third most common tumor in childhood and adolescence (1).

Correspondence to: Professor Shan Hu Huang, Department of Orthopedics, First Affiliated Hospital of Nanchang University, Nanchang, Jiangxi 330006, P.R. China

E-mail: hsh869@126.com

*Contributed equally

Key words: osteosarcoma, fatty acid synthase, immunohistochemical, metastasis
A significant number of studies on OS were published during the 1950s and 1960s, revealing poor 5-year survival rates of less than $15 \%$ (2). It was not until the early 1970s that the introduction of doxorubicin and methotrexate with leucovorin showed promise in improving the survival rates. Following the discovery of effective chemotherapy, the 5-year survival rates for patients treated with intensive multidrug chemotherapy and aggressive local control have been reported to be $55-80 \%$ (3-5); however, an improvement in survival rates has been limited only to the patients with a high grade of disease (5). Unfortunately, patients with metastatic disease have a poor prognosis, particularly those with pulmonary metastases at diagnosis, with various studies reporting 5-year survival rates of only $17-23 \%(6-8)$. Therefore, it is necessary to determine the mechanisms contributing to the metastasis of OS. Although many molecular factors linked to metastasis have been identified, a significant number still need to be investigated to provide a new therapeutic target for metastatic diseases in OS.

Fatty acid synthase (FASN) is an enzyme crucial for endogenous lipogenesis in mammals and is responsible for catalyzing the synthesis of long-chain fatty acids. FASN is expressed at high levels in a variety of human tumors (9-16), but shows low expression levels in normal tissues. This overexpression in neoplastic tissues makes FASN a potential diagnostic tumor marker. The inhibition of FASN activity is selectively cytotoxic to human cancer cells in vitro and in vivo $(17,18)$. Thus, FASN is considered a novel and promising target for antineoplastic therapy. Recent studies revealed that the inhibition of FASN promotes apoptosis and reduces cell growth and lymph node metastasis in a mouse melanoma model (19). In Colon 26 and CMT 93 cells, FASN contributed to metastasis by upregulating the activity of the AKT pathway (20). However, the expression of FASN and the correlation between FASN and metastasis in OS is still uncertain.

In the present study, we investigated FASN and Ki-67 protein expression levels in OS metastases using immunohistochemistry (IHC). We evaluated the correlation between FASN and Ki-67 expression levels in OS. Furthermore, the correlation between the expression levels of FASN and Ki-67 in OS tissues and OS metastasis was evaluated. Our findings revealed that there was a significant correlation between FASN, and Ki-67 expression levels and OS metastasis, and between 
Table I. FASN and Ki-67 protein expression levels in osteosarcoma and osteoenchondroma.

FASN expression levels

\begin{tabular}{lcrrrr}
\cline { 3 - 5 } Group (n) & Ki-67 label index & - & + & ++ & +++ \\
\hline Osteoenchondroma (21) & $2.05 \pm 0.86$ & 15 & 5 & 1 & 0 \\
Osteosarcoma (136) & $31.24 \pm 11.57$ & 50 & 18 & 24 & 44 \\
With metastasis (44) $^{\mathrm{b}}$ & $43.43 \pm 10.05$ & 6 & 8 & 12 & 18 \\
Without metastasis (92) $^{\mathrm{b}}$ & $25.41 \pm 6.68$ & 44 & 10 & 12 & 26
\end{tabular}

${ }^{\mathrm{a}} \mathrm{p}<0.05$ vs. osteoenchondronma group; ${ }^{\mathrm{b}} \mathrm{p}<0.05$ vs. without metastasis group. FASN, fatty acid synthase.

FASN and Ki-67 protein expression levels. This suggests that FASN may be involved in OS metastasis and may be a promising target for treating OS metastasis.

\section{Materials and methods}

Patients and tumor specimens. From 2005 to 2009, 136 patients with histologically proven OS of the extremities were treated at the First Affiliated Hospital of Nanchang University, China. There were 44 cases with pulmonary metastases and 92 cases without metastases at diagnosis. All of the specimens were obtained by excision biopsy. The analysis of pulmonary metastases was performed with CT scans at the time of the first diagnosis. No patients had a history of prior therapies with anticancer drugs or radiotherapy. The samples were fixed with $10 \%$ formalin, embedded in paraffin and then cut into $2-\mu \mathrm{m}$ sections. Twenty-one osteoenchondroma tissue samples were used as controls. The ethics committee of the First Affiliated Hospital of Nanchang University approved the study, and patients provided informed consent.

Immunohistochemistry for Ki-67 and FASN. Histological sections were cut at $2 \mu \mathrm{m}$ and stained with hematoxylin and eosin (H\&E). These sections underwent immunohistochemical analysis which was performed using the streptavidin-peroxidase (SP) procedure. Antigen retrieval was performed by heating the deparaffinized rehydrated sections in $10 \mathrm{~mm}$ citrate buffer ( $\mathrm{pH} 6.0$ for $20 \mathrm{~min}$ ) followed by blocking with $10 \%$ goat serum. The sections were subsequently incubated overnight at $4^{\circ} \mathrm{C}$ with the primary antibody (rabbit anti-FASN monoclonal antibody, Santa Cruz Biotechnology Inc., Santa Cruz, CA, USA; anti-Ki-67, Dako North America, Carpinteria, CA, USA). For the negative controls, the sections were incubated with phosphate-buffered saline (PBS) instead of antibodies. After washing with PBS three times, the sections were incubated with the biotinylated secondary antibody for $40 \mathrm{~min}$, followed by incubation with horseradish peroxidase (HRP)conjugated streptavidin for $30 \mathrm{~min}$. Finally, the sections were chemiluminescence-stained and counterstained using H\&E. The immunostaining was evaluated by two independent pathologists without knowledge of the clinical and pathological parameters.

Quantitative method. The expression levels of FASN were determined according to the staining intensity of at least 500 cells in 5 representative areas. Their intensity scores were recorded as: no staining (0); weak staining (1); moderate staining (2) and intense staining (3). The percentage scores were recorded as: less than $1 \%$ (score 0), less than $10 \%$ (score 1), 11 to $50 \%$ (score 2), 51 to $80 \%$ (score 3) and 81 to $100 \%$ (score 4). The final score was averaged with the scores from the two pathologists, which were calculated by the addition of the intensity score to the percentage score. The sections with a final score of less than 4 were considered as (-), score 4 was considered as (+), score 5 as $(++)$ and more than 6 was considered as $(+++)$. In all cases, brown staining in the cytoplasm was adopted as the standard for positivity.

For Ki-67 expression, a minimum of 400 cells were counted on each slide at a magnification of $x 400$, irrespective of whether they had been stained by anti-Ki-67 or not. In each case, 3 visual fields were evaluated. The percentage of stained cells for each case was obtained from the ratio between the number of cells with stained nuclei and unstained nuclei multiplied by 100 (label index).

Statistical analysis. All analyses were performed using the Statistical Package for the Social Sciences (SPSS Version 13.0, Chicago, IL, USA). Two independent samples were used to analyze the difference between FASN expression levels in OS and osteoenchondroma tissues, and between OS patients with and without pulmonary metastasis. An independent samples t-test was used to analyze the difference between Ki-67 expression levels in OS and in osteoenchondroma tissues, and between OS patients with and without pulmonary metastasis. The correlation of FASN with Ki-67 protein in OS tissues was evaluated using Spearman's rho. A value of $\mathrm{p}<0.05$ was considered to indicate a statistically significant result.

\section{Results}

FASN and Ki-67 protein expression in OS and osteoenchondroma. FASN protein was expressed in the cytoplasm and Ki-67 was expressed in the nuclei of OS and osteoenchondroma tissues (Fig. 1). The FASN positive expression rate was significantly higher in OS tissues (63.2\%) than in osteoenchondroma tissues (28.6\%) (Table I). The Ki-67 protein expression level was significantly higher in OS tissues than in osteoenchondroma tissues (Table I). The statistical analyses revealed a significant positive correlation between FASN and 

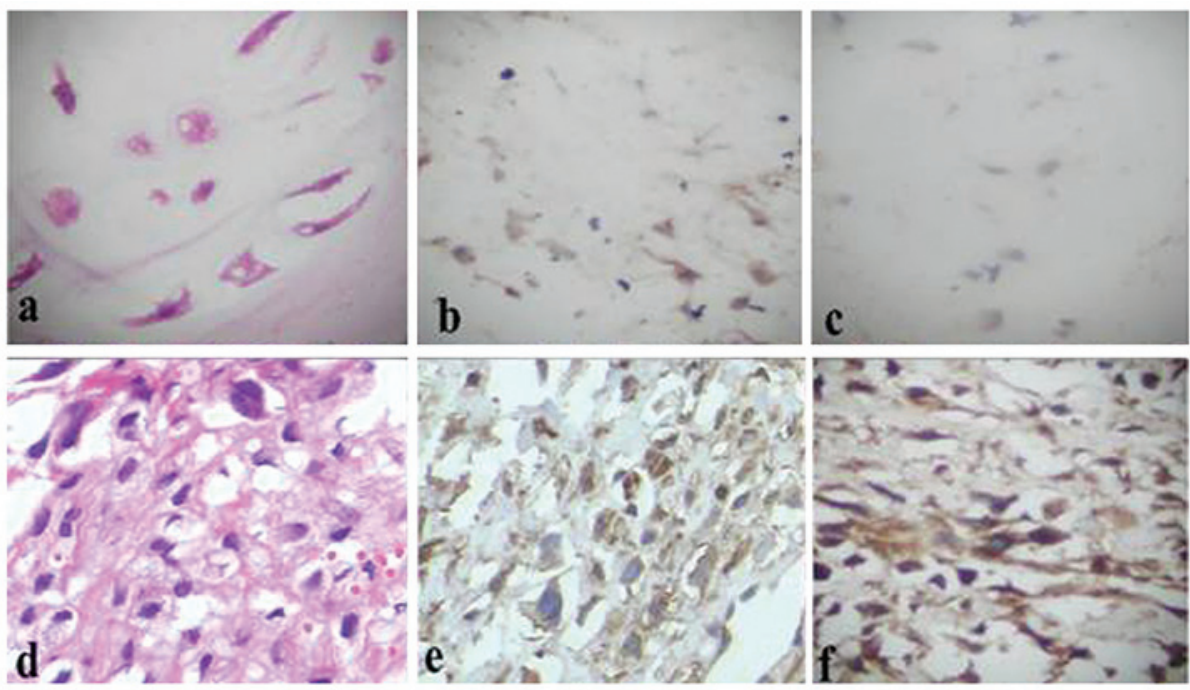

Figure 1. Fatty acid synthase (FASN) and Ki-67 expression in osteosarcoma (OS) and osteoenchondroma tissues (x400). Representative images of (a) H\&E staining for osteoenchondroma tissues; (b) IHC staining for FASN in osteoenchondroma tissues; (c) IHC staining for Ki-67 in osteoenchondroma tissues; (d) H\&E staining for OS tissues; (e) IHC staining for FASN in OS tissues; (f) IHC staining for Ki-67 in OS tissues. H\&E, hematoxylin and eosin; IHC, immunohistochemistry.
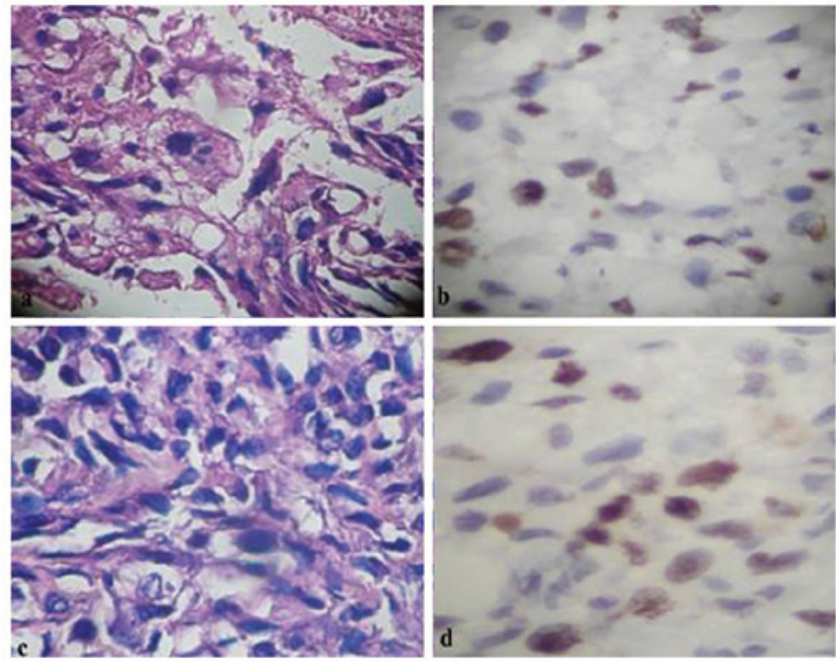

Figure 2. Ki-67 expression in osteosarcoma (OS) with and without pulmonary metastasis (x400). Representative images of (a) H\&E staining for OS tissues without pulmonary metastasis; (b) IHC staining for Ki-67 in OS tissues without pulmonary metastasis; (c) H\&E staining for OS tissues with pulmonary metastasis and (d) IHC staining for Ki-67 with pulmonary metastasis. H\&E, hematoxylin and eosin; IHC, immunohistochemistry.

Ki-67 protein expression (Table II). Our results indicated that FASN and Ki-67 are overexpressed in the tissues of OS.

The correlation between FASN, Ki-67 protein expression levels and pulmonary metastasis in OS. Under light microscopy, there was a greater concentration of Ki-67-stained nuclei in the cases with pulmonary metastasis compared to those without (Fig. 2). The Ki-67 proliferation index was significantly higher in OS tissues with pulmonary metastasis than in those without $(\mathrm{p}<0.05$, Table I). Thirty-eight cases $(86.4 \%)$ with pulmonary metastases at diagnosis were positive for FASN expression in the OS tissues, but only 48 cases
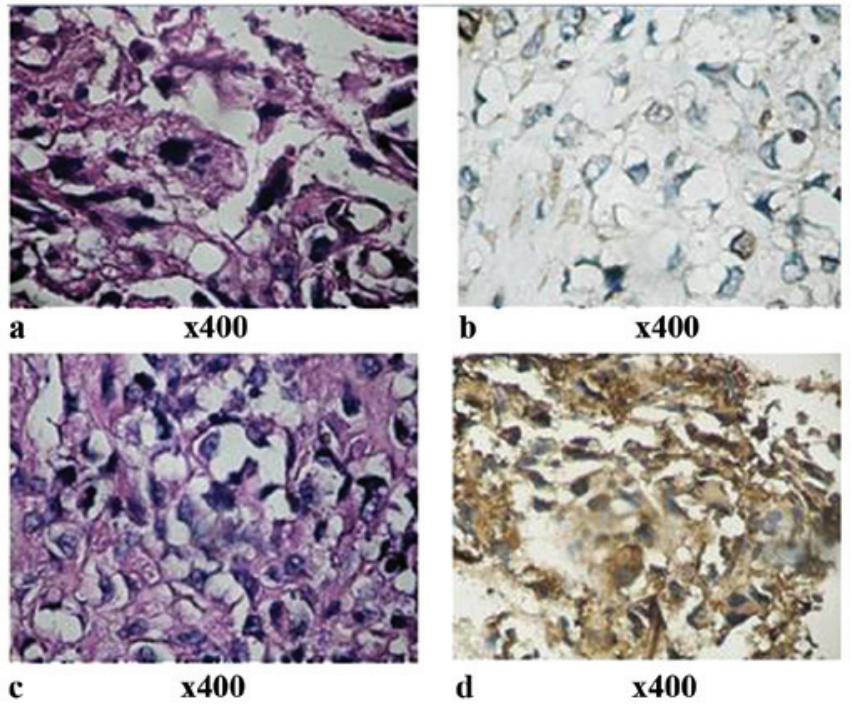

Figure 3. Fatty acid synthase (FASN) expression in osteosarcoma (OS) with and without pulmonary metastasis (x400). Representative images of (a) H\&E staining for OS tissues without pulmonary metastasis showing that OS is cell-rich and has significant cellular atypia, ansionucleosi, prominent nucleoli, abundant cytoplasm and a small amount of bone-like matrix; (b) IHC staining for FASN in OS tissues without pulmonary metastasis, showing yellow particle deposition in the cytoplasm with only a small amount of the cells, approximately $10 \%$, colored; (c) H\&E staining for OS tissues with pulmonary metastasis showing that OS is cell-rich and has significant cellular atypia, ansionucleosi, prominent nucleoli and abundant cytoplasm; (d) IHC staining for FASN with lung metastasis showing brown particles deposited in the cytoplasm with the majority of cells, approximately $92 \%$, colored. H\&E, hematoxylin and eosin; IHC, immunohistochemistry.

$(52.2 \%)$ of those without pulmonary metastases were positive (Table I). There were also more cells with a positive expression for FASN protein in the cases with pulmonary metastasis, as shown by an intensely stained cytoplasm, compared to the cases without pulmonary metastasis (Fig. 3). The expression level was significantly higher in the cases with lung metastasis 
Table II. Correlation between FASN ans Ki-67 protein expression levels in osteosarcoma.

\begin{tabular}{lcc}
\hline FASN expression levels & No. of cases & Ki-67 label index \\
\hline- & 50 & $22.38 \pm 4.28$ \\
+ & 18 & $30.22 \pm 8.32$ \\
++ & 24 & $35.41 \pm 10.37$ \\
+++ & 44 & $41.68 \pm 10.49$ \\
\hline
\end{tabular}

FASN, fatty acid synthase; Spearman's rho, F=43.05, R=0.734.

than in the cases without metastasis $(\mathrm{p}<0.01)$. Our data suggest that FASN and Ki-67 may be involved in OS metastasis.

\section{Discussion}

$\mathrm{Ki}-67$ is a type of nuclear protein which is expressed in the $\mathrm{G} 1, \mathrm{~S}, \mathrm{G} 2$ and $\mathrm{M}$ phases of the cellular cycle, but not in the G0 phase. Ki-67 protein is present in all mitogenic cells of normal and tumor tissues. Ki-67 protein expression levels not only reflect the activity of tumor cells, but are also correlated with the development, metastasis and prognosis of cancer. The present study evaluated the proliferation of Ki-67 in OS and osteoenchondroma tissues and the results revealed that the percentage of positively stained cells for Ki-67 protein expression was $31.24 \pm 11.57$ and $2.05 \pm 0.86 \%$ in OS and osteoenchondroma tissues, respectively $(\mathrm{p}<0.01)$. The expression levels of $\mathrm{Ki}-67$ protein in OS were significantly higher than in osteoenchondroma tissues. Furthermore, the correlation between Ki-67 protein expression levels and pulmonary metastasis was investigated in this study and the results showed a significant correlation between Ki-67 protein expression levels and pulmonary metastasis of OS $(\mathrm{p}<0.01)$, which was consistent with the results from a previous study (21). The results suggest that $\mathrm{Ki}-67$ protein may be involved in OS metastasis.

Fatty acid metabolic pathways have an important role in carcinogenesis (22). Human FASN is a $270 \mathrm{kDa}$ cytosolic dimeric enzyme that is responsible for fatty acid synthesis. Endogenous fatty acid synthesis from the small carbon precursors acetyl-CoA and malonyl-CoA is dependent on the activity of FASN. FASN is downregulated by dietary fatty acids in the majority of cells, with the exception of lipogenic tissues such as liver, lactating breast, fetal lung and adipose tissues. Recent studies have provided compelling evidence that neoplastic lipogenesis is essential for cancer cell survival (23). Various studies have reported FASN overexpression in a variety of human tumors (24-29). In this study, we demonstrated that FASN was positively expressed in 86 of 136 OS cases (63.2\%); however, the rate of positive expression was $28.6 \%$ in osteoenchondroma tissues. The difference in FASN expression level between OS and osteoenchondroma was significant $(\mathrm{p}<0.01)$. Recent studies have shown that FASN expression levels are associated with tumor cells metastasis in vivo $(20,30)$. In our study, FASN was overexpressed in 38 of 44 patients with pulmonary metastasis, but only 48 of the 92 patients without metastasis demonstrated positive FASN expression.
The difference between expression levels of FASN in cases with pulmonary metastasis was significantly higher than in those without metastasis ( $\mathrm{p}=0.002$ ), showing similar results to previous studies. This suggests that FASN may be involved in OS metastasis.

As the correlation between $\mathrm{Ki}-67$ protein and OS metastasis has been revealed in previous studies, we investigated the correlation of FASN and Ki-67 protein expression levels in OS tissue to indirectly provide evidence of the correlation between FASN and OS metastasis. Our results revealed a positive correlation between FASN and Ki-67 protein expression levels and therefore we believe that FASN may be involved in OS metastasis.

To our knowledge, this is the first study to evaluate the correlation between FASN expression levels and metastasis of OS. Although further studies are required, the present findings show that FASN may be involved in proliferation and metastasis of OS, thereby possibly suggesting FASN as a promising target for treating OS metastasis.

\section{Acknowledgements}

This study was supported by Grants-in-Aid from the Technology Department, Education Office of Jiangxi Province (No. GJJ11316). We would like to thank associate pathologist Dr Li Weihua (Yuyao People's Hospital, Zhejiang, China) for the immunostaining evaluation.

\section{References}

1. Longhi A, Errani C, De Paolis M, et al: Primary bone OS in the pediatric age: State of the art. Cancer Treatment Reviews 32: 423-436, 2006.

2. Mckenna R, Schwinn CP, Soong KY, et al: Sarcomata of the osteogenic series (osteosarcoma, fibrosarcoma, chondrosarcoma, parosteal osteogenic sarcoma, and sarcomata arising in abnormal bone) an analysis of 552 cases. J Bone Joint Surg 48: 1-26, 1966.

3. Iwamoto Y, Tanaka K, Isu K, et al: Multiinstitutional phase II study of neoadjuvant chemotherapy for OS (NECO study) in Japan: NECO-93J and NECO-95J. J Orthop Sci 14: 397-404, 2009.

4. Meyers PA, Schwartz CL, Krailo M, et al: OS: A randomized, prospective trial of the addition of ifosfamide and/or muramyl tripeptide to cisplatin, doxorubicin, and high-dose methotrexate. J Clin Oncol 23: 2004-2011, 2005.

5. Jawad MU, Cheung MC, et al: Osteosarcoma: improvement in survival limited to high-grade patients only. J Cancer Res Clin Oncol 137: 597-607, 2011.

6. Mialou V, Philip T, Kalifa C, et al : Metastatic OS at diagnosis: prognostic factors and long-term outcome - the French pediatric experience. Cancer 104: 1100-1109, 2005.

7. Hegyi M, Semsei AF, Jakab Z, et al: Good prognosis of localized $\mathrm{OS}$ in young patients treated with limb-salvage surgery and chemotherapy. Pediatr Blood Cancer 57: 415-422, 2011.

8. Stokkel MP, Linthorst MF, Borm JJ, et al: A reassessment of bone scintigraphy and commonly tested pretreatment biochemical parameters in newly diagnosed osteosarcoma. J Cancer Res Clin Oncol 128: 393-399, 2002.

9. Alo PL, Amini M, Piro F, et al: Immunohistochemical expression and prognostic significance of fatty acid synthase in pancreatic carcinoma. Anticancer Res 27: 2523-2527, 2007.

10. Kusakabe T, Nashimoto A, Honma K, et al: Fatty acid synthase is highly expressed in carcinoma, adenoma and in regenerative epithelium and intestinal metaplasia of the stomach. Histopathology 40: 71-79, 2002.

11. Walter K, Hong SM, Nyhan S, et al: Serum fatty acid synthase as a marker of pancreatic neoplasia. Cancer Epidemiol Biomarkers Prev 18: 2380-2385, 2009.

12. Okawa Y, Hideshima T, Ikeda H, et al: Fatty acid synthase is a novel therapeutic target in multiple myeloma. British Journal of Haematology 141: 659-671, 2008. 
13. Migita T, Ruiz S, Fornari A, et al: Fatty acid synthase: a metabolic enzyme and candidate oncogene in prostate cancer. J Natl Cancer Inst 101: 519-532, 2009.

14. Yan S, Kefeng W and Sheng SY: Expression of fatty acid synthase in bladder transitional cell carcinoma and its significance. China Oncology 17: 395-397, 2007.

15. Silva SD, Cunha IW, Younes RN, et al: ErbB receptors and fatty acid synthase expression in aggressive head and neck squamous cell carcinomas. Oral Dis 16: 774-780, 2010.

16. Crispino P, Alò PL, Rivera M, et al: Evaluation of fatty acid synthase expressionin oesophageal mucosa of patients with oesophagitis, Barrett's oesophagus and adenocarcinoma. J Cancer Res Clin Oncol 135: 1533-1541, 2009.

17. Orita H, Coulter J, Tully E, et al: Inhibiting fatty acid synthase for chemoprevention of chemically induced lung tumors. Clin Cancer Res 14: 2458-2464, 2008.

18. Coleman DT, Bigelow R and Cardelli JA: Inhibition of fatty acid synthase by luteolin post-transcriptionally down-regulates c-Met expression independent of proteosomal lysosomal degradation. Mol Cancer Ther 8: 214-224, 2009.

19. Carvalho MA, Zecchin KG, Seguin F, et al: Fatty acid synthase inhibition with Orlistat promotes apoptosis and reduces cell growth and lymph node metastasis in a mouse melanoma model. Int J Cancer 123: 2557-2565, 2008.

20. Murata S, Yanagisawa K, Fukunaga K, et al: Fatty acid synthase inhibitor cerulenin suppresses liver metastasis of colon cancer in mice. Cancer Sci 101: 1861-1865, 2010.

21. Lin C, Ming LW, Bao LF, et al: Expressions of P21(WAF1) protein, CNA and Ki-67 and their significance in osteosarcoma. China Oncology 11: 109-112, 2001.

22. Yeh CS, Wang JY, Cheng TL, et al: Fatty acid metabolism pathway play an important role in carcinogenesis of human colorectal cancers by Microarray Bioinformatics analysis. Cancer Lett 233: 297-308, 2006.
23. Flavin R, Peluso S, Nguyen PL and Loda M: Fatty acid synthase as a potential therapeutic target in cancer. Future Oncol 6: 551-562, 2012.

24. Takahiro T, Shinichi K and Toshimitsu S: Expression of fatty acid synthase as a prognostic indicator in soft tissue sarcomas. Clin Cancer Res 9: 2204-2212, 2003.

25. Kusakabe T, Maeda M, Hoshi N, et al: Fatty acid synthase is expressed mainly in adult hormone-sensitive cells or cells with high lipid metabolism and in proliferating fetal cells. J Histochem Cytochem 48: 613-622, 2000.

26. Menendez JA and Lupu R: Fatty acid synthase and the lipogenic phenotype in cancer pathogenesis. Nat Rev Cancer 7: 763-777, 2007.

27. Rossi S, Ou W, Tang D, et al: Gastrointestinal stromal tumours overexpress fatty acid synthase. J Pathol 209: 369-375, 2006.

28. Silva SD, Perez DE, Nishimoto IN, et al: Fatty acid synthase expression in squamous cell carcinoma of the tongue: clinicopathological findings. Oral Dis 14: 376-382, 2008.

29. Alò PL, Visca P, Framarino ML, et al: Immunohistochemical study of fatty acid synthase in ovarian neoplasms. Oncol Rep 7: 1383-1388, 2000.

30. Selvendiran K, Ahmed S, Dayton A, et al: HO-3867, a synthetic compound, inhibits the migration and invasion of ovarian carcinoma cells through downregulation of fatty acid synthase and focal adhesion kinase. Mol Cancer Res 8: 1188-1197, 2010. 